\title{
COMPORTAMENTO DE CULTIVARES DE ALFACE EM FUNÇÃO DO ESPAÇAMENTO
}

\author{
Márcia De Moraes Echer ${ }^{2}$ \\ José Maria M. Sigrist ${ }^{1}$ \\ Vandeir Francisco Guimarães ${ }^{2}$ \\ Keigo Minami ${ }^{3}$
}

\section{RESUMO}

Este trabalho foi conduzido na área experimental do Departamento de Produção Vegetal/ESALQ, Piracicaba-SP, no período de setembro a dezembro de 1998, com o objetivo de avaliar o comportamento de cinco cultivares de alface (Lactuca sativa $\mathrm{L}$ ) em dois espaçamentos. Os tratamentos foram um fatorial de cinco cultivares de alface crespa: (Brisa, Grande Rápida, Marisa, Vera e Verônica AF 257) e dois espaçamentos: $(0,20 \times 0,20 \mathrm{~m}$ e $0,25 \times 0,25 \mathrm{~m})$. Usaram-se quatro blocos casualizados. Foram avaliados os seguintes parâmetros: Massa fresca da parte aérea por planta, número de folhas por planta, massa fresca foliar por planta, massa fresca média de uma folha, relação entre a massa fresca foliar por planta e massa fresca de parte aérea por planta e produção média por unidade de área. Não houve interação significativa entre cultivares e espaçamentos para os parâmetros avaliados. De maneira geral, as cultivares Vera, Marisa e Brisa se destacaram. Obteve-se maior produção por unidade de área no menor espaçamento com plantas dentro de padrões comerciais. O cultivar Vera apresentou maior relação entre massa fresca foliar

1 Pós-Graduando do Dep. de Produção Vegetal - ESALQ/USP, C.P. 09, CEP: 13.418-900, Piracicaba, SP.

2 Pós-Graduando do Dep. de Produção Vegetal - ESALQ/USP, C.P. 09, CEP: 13.418-900, Piracicaba, SP. 2Pós-Graduando do Dep. de Produção Vegetal, FCA/ UNESP, CP: 237, CEP:18.603-970, Botucatu, SP.

3 Dep. de Produção Vegetal - ESALQ/USP. 
por planta e massa fresca da parte aérea por planta, o que é vantajoso.

Palavras-chave: Lactuca sativa $\mathrm{L}$, alface crespa, densidade de plantio, produção.

\section{BEHAVIOR OF LETTUCE CULTIVARS IN FUNCTION OF SPACINGS}

\section{ABSTRACT}

This study was carried out at the Department of Vegetable Production (ESALQ, Piracicaba-SP) from September to December 1998, to evaluate the performance of five lettuce cultivars in two spacings. The treatments were a factorial of five crisp lettuce cultivars: (Brisa, Grande Rápida, Marisa, Vera e Verônica Af 257) x two spacings: $0,20 \times 0,25 \mathrm{~m}$ and $0,25 \times 0,25 \mathrm{~m}$ in four randomized blocks. The following parameteres were evaluated: fresh matter of aerial part per plant, number of leaves per plant, leaf fresh matter per plant, average fresh matter of one leaf, relation between leaf fresh matter per plant and fresh matter of aerial part per plant, and average total production per area. No significant interaction between cultivars and spacings was found. In general, the best cultivars were Vera, Marisa and Brisa. In the small spacing there was a higher production per area with plant within comercial standards. Cultivar Vera presented higher relation between the fresh matter of leaves per plant and the fresh matter of aerial part per plant, an advantageous characteristic.

Key words: Lactuca sativa L, crisp lettuce, plant density, production.

\section{INTRODUÇÃO}

A alface (Lactuca sativa L.) é a principal hortaliça do grupo das folhosas cultivada no Brasil. Hoje mundialmente conhecida e utilizada em saladas e sanduíches, é excelente fonte de vitamina A, com quantidades apreciáveis de vitaminas B1 e B2, além de vitamina C. 
Os diferentes cultivares de alface hoje disponíveis para comercialização originaram-se de sucessivas seleções artificiais (Davis et al., 1997). Estas são agrupadas, considerando-se as características das folhas, bem como o fato de formarem ou não cabeça repolhuda, obtendose assim seis tipos: repolhuda manteiga, repolhuda crespa (Americana), solta lisa, solta crespa, mimosa e romana (Filgueira, 2000).

Grande parte da alface produzida pertence ao tipo manteiga, que tem preferência predominante em todo o Brasil, porém, nos últimos anos, com o crescimento da aceitação da alface de folha crespa, as áreas de produção desta têm aumentado significativamente (Nagai, 1993), além do que ela é mais produtiva nas condições de primavera-verão (Nicoulaud \& Porto, 1990). Embora existam cultivares adaptados ao verão, é indispensável testá-los nas condições locais, pois seu comportamento pode variar em função das condições climáticas.

Sabe-se que o espaçamento entre fileiras e entre plantas exerce grande influência no comportamento das plantas, afetando-lhes a arquitetura, desenvolvimento, peso, qualidade, e, dentre outras características, a mais importante a produção, que resulta da interação do genótipo das plantas com as condições ambientais. Entre os fatores que afetam a condição do meio está a densidade de plantio (Arismedi, 1975).

Maciel (1968) relata que para o cultivo da alface o espaçamento exerce influência altamente significativa, quanto ao número de plantas obtidas na colheita, na produção total e na porcentagem de plantas comerciais. Já Carneiro (1981) observou que nas densidades mais baixas, o peso médio e o valor comercial mostraram-se inferiores aqueles obtidos nas densidades médias. Segundo Mondin et al. (1989), a diminuição na densidade de plantio permitiu um acréscimo no peso e diâmetro médio de cabeças.

O presente trabalho teve como objetivo avaliar o comportamento de cinco cultivares de alface (Lactuca sativa L) em duas densidades de plantio.

\section{MATERIAL E MÉTODOS}

O experimento foi conduzido no Campo Experimental do Setor 
de Horticultura, do Departamento de Produção Vegetal da Escola Superior de Agricultura Luiz de Queiroz (ESALQ) em Piracicaba-SP, no período de setembro a dezembro de 1998. Usou-se um fatorial de cinco cultivares de alface: (Vera) (Agroflora), Marisa (Agroceres), Brisa (Asgrow), Crespa Grande Rápida (Agroflora) e Crespa Verônica-AF.257 (Agroflora) $x$ dois espaçamentos: $0,20 \times 0,20 \mathrm{~m}$ e $0,25 \times 0,25 \mathrm{~m}$, em quatro blocos casualizados

Em setembro de 1998, sementes nuas dos cinco cultivares foram semeadas em bandejas de poliestireno expandido, com 288 células, em substrato artificial, (Gioplant III). As mudas foram transplantadas para condições de campo quando apresentavam 4 a 6 folhas definitivas. Os tratos culturais foram realizados de acordo com a recomendação para a cultura sendo a adubação baseada nos resultados da análise de solo.

As parcelas apresentavam áreas de 1,20 e $1,25 \mathrm{~m}^{2}$ para os espaçamentos de $0,20 \times 0,20 \mathrm{~m} \mathrm{e} 0,25 \times 0,25 \mathrm{~m}$ respectivamente, resultando em parcelas com 30 plantas para o primeiro espaçamento e 20 para o segundo. A colheita foi realizada 69 dias após a semeadura, quando as plantas alcançaram o máximo crescimento vegetativo, sendo colhidas em cada parcela 6 plantas, correspondentes a uma área útil de 0,2400 e $0,3750 \mathrm{~m}^{2}$ para os dois espaçamentos respectivamente. Avaliaram-se as seguintes variáveis: massa fresca da parte aérea por planta em g (MFPA), número de folhas por planta (NF), massa fresca foliar por planta em $g$ (MFF), massa fresca média de uma folha em $\mathrm{g}$ (MFMF), relação entre massa fresca foliar por planta e massa fresca da parte aérea por planta (RFPA) e produção média por unidade de área em t/ha (PM). A produção total foi calculada considerando-se a perda de $1 / 4$ da área ocupada por caminhos e espaços entre canteiros.

Os dados obtidos foram submetidos à análise de variância, e as médias comparadas através do teste de Tukey a $5 \%$ de probabilidade.

\section{RESULTADOS E DISCUSSÃO}

Observou-se pelo resultado da análise de variância resumida na Tabela 1, que não houve interação significativa entre cultivar e 
espaçamento para nenhuma das variáveis avaliadas. Os cultivares mantiveram comportamento semelhante nas duas densidades de plantio, confirmado pela falta de interação entre os cultivares e o espaçamento, mostrado na Tabela 1. Porém, diferenças estatísticas significativas foram observadas entre cultivares e entre espaçamentos.

A Tabela 2 apresenta os resultados referentes ao teste de médias para cultivares e espaçamentos nos diferentes parâmetros avaliados. De maneira geral, os cultivares Vera, Marisa e Brisa se destacaram. Porém para a RFPA notou-se um comportamento diferenciado, destacando-se o cultivar Vera, seguido pelo Verônica AF257. O cultivar Verônica AF257 mostrou comportamento intermediário para a maioria dos parâmetros, em comparação com os demais.

O cultivar Vera mostrou-se inferior quanto ao NF em comparação ao Brisa. Este dado resultou em maior MFMF para este cultivar. Para o parâmetro RFPA o cultivar Vera foi o que se destacou, porém não diferiu estatisticamente dos cultivares Verônica e Marisa. Esta é uma característica de grande importância, indicativa de que a planta tem maior porção comestível (folha) em relação à não comestível (caule). Este cultivar se destacou na produção por maior MFMF. O cultivar Grande Rápido foi inferior aos demais em todos os parâmetros avaliados.

Entre os cultivares considerados promissores, destacam-se Marisa, Brisa e Vera, com produção de 36,21; 35,77 e 33,08 t/ha respectivamente. Azevedo (1997) relata que na época de outubro/novembro, no Estado de Tocantins, os cultivares Verônica e Marisa mostraram produtividades de 45 e 44 t/ha respectivamente. Resultados semelhantes foram encontrados por Lédo et al. (1998), para o Estado do Acre. No entanto os autores não mencionam a perda de área útil ocupada por espaços entre canteiros e caminhos, o que pode explicar as diferenças de produtividade encontradas.

Ainda na Tabela 2, nota-se a superioridade da densidade $0,25 \mathrm{x}$ 0,25m para MFPA, MFF e NF. De acordo com Maciel (1968) e Mondin et al. (1989), o efeito do espaçamento, no comportamento de cultivares de alface, é atribuído à menor competição pelas plantas por luz, água e 
Tabela 1. Quadrado médio dos tratamentos para os diferentes parâmetros analisados. Massa fresca da parte aérea (MFPA), massa fresca foliar (MFF), massa fresca média de uma folha (MFMF), número de folha (NF), relação entre massa fresca foliar por planta e massa fresca da parte aérea por planta (RFPA) e produção média por unidade de área em tha (PM).

\begin{tabular}{|c|c|c|c|c|c|c|c|}
\hline \multirow{2}{*}{$\begin{array}{l}\text { Fonte de } \\
\text { variação }\end{array}$} & \multirow[b]{2}{*}{ GL } & \multicolumn{6}{|c|}{ Quadrado Médio } \\
\hline & & MFPA (g) & $\operatorname{MFF}(\mathrm{g})$ & MFMF (g) & NF & RFPA & PM (t/ha) \\
\hline Blocos & 3 & $3574,25^{\text {n }}$ & $1351,38^{\mathrm{nB}}$ & $0,39^{\mathrm{ns}}$ & $38,84^{* *}$ & $0,0043^{*}$ & $66,56 \mathrm{~ns}$ \\
\hline Cultivares (C) & 4 & $7424,83^{\circ "}$ & $5675,48^{* *}$ & $6,65^{* *}$ & $92,51^{\cdots *}$ & $0,0086^{* 4}$ & $161,52^{* *}$ \\
\hline Espaçamento (E) & 1 & $6745,49^{\circ}$ & $3464,34^{*}$ & $0,57^{\mathrm{ne}}$ & $35,16^{\circ}$ & $0,0007^{\mathrm{ns}}$ & $1058,84^{\circ *}$ \\
\hline Interação C x E & 4 & $1132,42^{n s}$ & $773,38^{\text {กв }}$ & $1,12^{\mathrm{ng}}$ & $2,42^{\mathrm{nB}}$ & $0,0014^{\mathrm{ns}}$ & $19,94^{\text {nв }}$ \\
\hline Resíduo & 27 & 1394,80 & 699,02 & 0,62 & 8,30 & 0,0010 & 27,24 \\
\hline $\mathbf{C V}$ & $\cdots$ & $17,65 \%$ & $15,65 \%$ & $10,91 \%$ & $12,29 \%$ & $3,96 \%$ & $16,26 \%$ \\
\hline
\end{tabular}

$\left({ }^{* *}\right)$ Significativo a $1 \%$ de probabilidade pelo teste F. $\left({ }^{*}\right)$ Significativo a $5 \%$ de probabilidade pelo teste $\mathrm{F}$.

(ns) Não significativo a $5 \%$ de probabilidade pelo teste $F$.

nutrientes. Para couve-flor, segundo Minami e Victória (1981), a competição entre plantas é muito grande, tendendo a produzir cabeças pequenas e reduzido número de folhas, sendo a produção total por hectare pouco afetada.

Quanto às variáveis MFMF e RFPA, mostraram-se insensíveis à densidade de plantio, não mostrando diferenças estatísticas para os dois espaçamentos testados. Para a MFMF este resultado pode ser explicado pelo efeito de compensação devido ao aumento do número de folhas por planta na menor densidade de plantio, ou seja maior espaçamento entre plantas. Quanto à RFPA, pode-se sugerir que seja uma característica genética pouco sensível às variações ambientais causadas pelas duas densidades de plantio utilizadas.

Analisando produção por unidade de área (PM), também na Tabela 2, nota-se comportamento inverso ao dos demais parâmetros, sendo 
Tabela 2. Médias para cinco cultivares e dois espaçamentos para os seguintes parâmetros: Massa fresca da parte aérea (MFPA), massa fresca foliar (MFF), massa fresca média de uma folha (MFMF), número de folhas (NF), relação entre massa fresca foliar por planta e massa fresca da parte aérea por planta (RFPA) e produção média por unidade de área em t/ha (PM). Piracicaba, ESALQ, 1998.

\begin{tabular}{|c|c|c|c|c|c|c|}
\hline Cultivares & MFPA (g) & $\operatorname{MFF}(\mathrm{g})$ & MFMF (g) & NF & RFPA & PM (t/ha) \\
\hline Brisa & $232,91 \mathrm{a}$ & 180,99 a & $6,41 \mathrm{bc}$ & $28,37 \mathrm{a}$ & $0,78 \mathrm{bc}$ & $35,77 \mathrm{a}$ \\
\hline Grande rápida & $165,34 \mathrm{~b}$ & $124,93 \mathrm{~b}$ & $6,28 \mathrm{c}$ & $19,87 \mathrm{c}$ & $0,76 \mathrm{c}$ & $25,38 \mathrm{~b}$ \\
\hline Marisa & $241,87 \mathrm{a}$ & $191,09 \mathbf{a}$ & $7,47 a b$ & $25,40 \mathrm{ab}$ & $0,80 \mathrm{abc}$ & $36,21 \mathrm{a}$ \\
\hline Vera & $218,65 \mathrm{ab}$ & $184,29 \mathrm{a}$ & $8,53 \mathrm{a}$ & $21,62 b c$ & $0,84 \mathrm{a}$ & $33,08 \mathrm{a}$ \\
\hline Verônica AF 257 & $199,25 \mathrm{ab}$ & $163,52 \mathrm{ab}$ & $7,27 \mathrm{bc}$ & $22,00 \mathrm{bc}$ & $0,82 a b$ & $30,03 \mathrm{ab}$ \\
\hline$\Delta$ & 54,59 & 38,65 & 1,15 & 4,21 & 0,05 & 7,63 \\
\hline Espaçamento & MFPA (g) & MFF (g) & MFMF (g) & NF & RFPA & PM (t/ha) \\
\hline $0,20 \times 0,20 \mathrm{~m}$ & $198,62 \mathrm{~B}$ & $159,66 \mathrm{~B}$ & $7,31 \mathrm{~A}$ & $22,52 \mathrm{~B}$ & $0,80 \mathrm{~A}$ & $37,24 \mathrm{~A}$ \\
\hline $0,25 \times 0,25 \mathrm{~m}$ & $224,59 \mathrm{~A}$ & $178,27 \mathrm{~A}$ & $7,07 \mathrm{~A}$ & $24,39 \mathrm{~A}$ & $0,80 \mathrm{~A}$ & $26,95 \mathrm{~B}$ \\
\hline
\end{tabular}

'Médias seguidas pela mesma letra na coluna não diferem pelo teste Tukey a $5 \%$ de probabilidade. $\Delta$-Diferença mínima significativa para médias de cultivares.

superior estatisticamente para a densidade de plantio $0,20 \times 0,20 \mathrm{~m}$. É importante notar que, apesar de a menor densidade de plantio ter resultado em plantas com maior MFP, na maior densidade ainda foram obtidas plantas com características comerciais. Estes dados mostram que, à medida que diminui o espaçamento e aumenta a densidade populacional, dentro de certos limites, há tendência de aumentar a produção total por área, podendo ser mais lucrativo para o produtor, embora nem sempre se obtenham produtos de alto valor comercial, conforme observado por Maciel (1968) e Arismed (1975). 


\section{CONCLUSÕES}

- Os cultivares Vera, Marisa e Brisa foram os que se destacaram nas condições de estudo. Porém, o cultivar Vera mostrou maior relação entre a massa fresca foliar por planta e massa fresca da parte aérea por planta, característica altamente desejável.

- A maior densidade de plantio mostrou-se mais vantajosa por apresentar maior produção de massa fresca por unidade de área, com plantas ainda dentro de padrões comerciais;

- A relação entre a massa fresca foliar e a massa fresca da parte aérea não foi sensível a mudanças na densidade de plantio, mostrando ser característica genética pouco variável em função da variação ambiental provocada pelos espaçamentos estudados.

\section{REFERÊNCIAS BIBLIOGRÁFICAS}

ARISMENDI, L.G., 1975. Efeito de Métodos de Produção de Mudas e População no Rendimento do Repolho (Brassica oleracea va. Capitata). Viçosa, 50p. (Dissertação de Mestrado) - Universidade Federal de Viçosa.

AZEVEDO, S.M.; MOMENTÉ, V.G.; SILVEIRA, M.A.; DE SÁ, M.; MALUF, W.R.; BLANK, A.F., 1997. Avaliação de Cultivares de Alface Para as Condições Quente e Úmida do Estado do Tocantins. In: CONGRESSO DE OLERICULTURA, 37., 1997, Manaus. Resumo... SOB, Manaus, 1997.

CARNEIRO, I.F., 1981. Competição Entre a Cultura do Repolho (Brassica oleracea var. capitata) e a Cultura da Alface (Lactuca sativa L.) em Cultivo Misto e em Diferentes Densidades de Populações. Piracicaba, 69p. (Dissertação de Mestrado). Escola Superior de Agricultura "Luiz de Queiroz", Universidade de São Paulo.

DAVIS, R.M.; SUBBARAO, K.V.; RAID, R.N.; KURTS, E.A., 1997. Compendium of Lettuce Disease, St. Paul. Press, 79p. FILGUEIRA, F.A.R., 2000, Novo Manual de Olericultura. Viçosa, 
UFV. 402p.

LÉDO, F.J. da S.; SOUSA, J.A. de.; SIVIERO, A.; SILVA, M.R. da.; ARAÚJO, H.M. de., 1998. Recomendação de Cultivares de Alface Para o Cultivo no Estado do Acre. Acre: EMBRAPA-CPAA, 1998. 2p. (EMBRAPA-CPAA, Comunicado Técnico, n.94).

MACIEL, R.F.P., 1968. Estudo Sobre a Influência do Espaçamento, Níveis de Irrigação na Cultura da Alface (Lactuca sativa L.). Viçosa. (Dissertação de Mestrado) - Universidade Federal de Viçosa. MINAMI, K.; VICTORIA, Fo., R., 1981. Efeito da Densidade de População de Plantas na Cultivar de Couve-Flor (Brassica oleracea var. botrytis). Anais da ESALQ, Piracicaba, v.28, p.1-10.

MONDIN, M.; ALVARENGA, M.A.R.; SOUZA, J.R.; VIEIRA, M.G.G.C., 1989. Influência de Espaçamentos, Métodos de Plantio de Sementes Nuas e Peletizadas na Produção de Duas Cultivares de Alface (Lactuca sativa L). Ciência e Prática, Lavras, v.13, n.2, p.185-194, 1989.

NAGAI, H., 1993. Alface Tipo Manteiga. In: FURLANI, A.M.C. \& VIÉGAS, G.P. eds. O Melhoramento de Plantas no Instituto Agronômico. Vol. 1. Campinas: Instituto Agronômico. 1993. p.204-221. NICOULAUD, A.L.; PORTO, M.D. de M., 1990. Desempenho de Oito Cultivares de Alface do Grupo Folha Lisa Durante a Primavera, no Sul do Brasil. Horticultura Brasileira, Brasília, v.8, n.2, p.30-31. 